Statics and Dynamics of Dimensionally and Spatially

\title{
Constrained Oxides
}

\author{
Summary Progress Report
}

Submitted to

Department of Energy

Basic Energy Science Division

Division of Materials Science \& Engineering

By

Vinayak P. Dravid

Professor, Materials Science \& Engineering

Director, NUANCE Center

Northwestern University

Evanston, IL 60208

Original Submission Date

March 2010
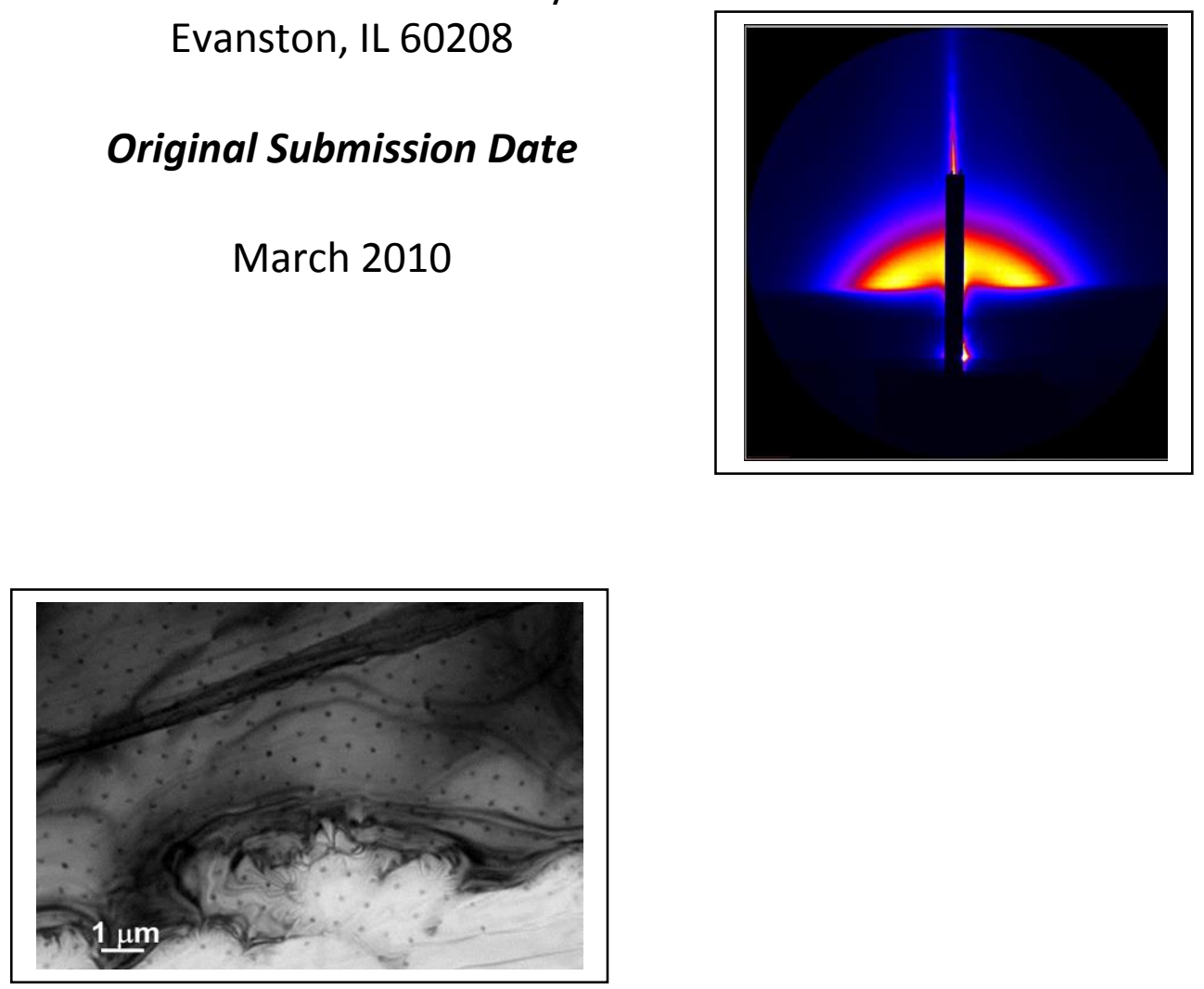


\section{Prior Research Accomplishments:}

Table I summarizes the various activities in the broad research program, proposed in 2006 (The various sub-themes that have been accomplished are noted in "tick marks").

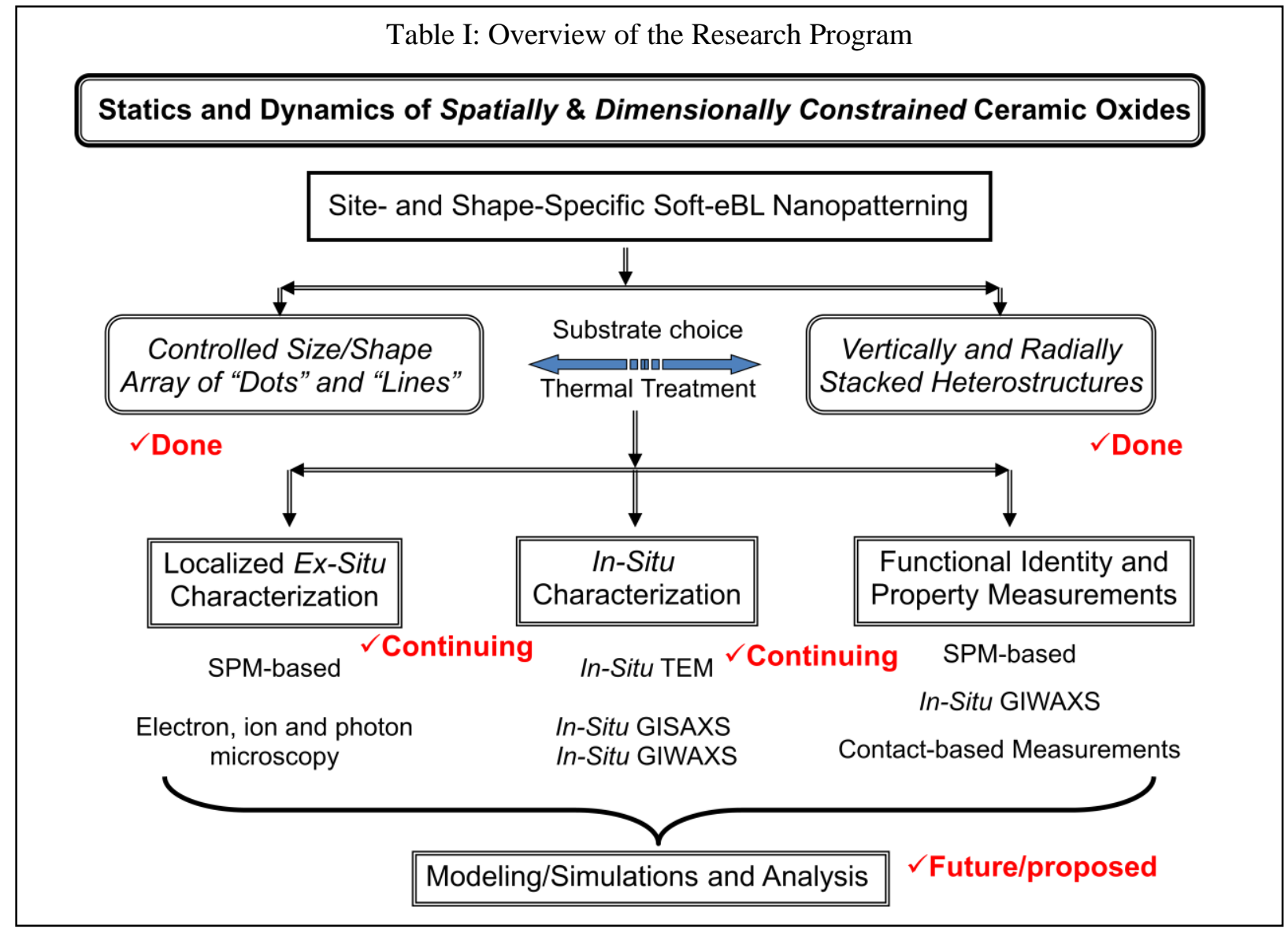

Specifically, the progress in prior research covered the following themes, including some of the highlights of prior accomplishments.

I.1: Development of soft eBL as an enabling approach for spatial and dimensional confinement of ceramic oxide nanostructures.

I.2. Optimization of thermal conversion of sol-gel to enable internal "microstructure" control, including innovative phase separation approach for coupled multiferroic phenomena.

I.3: Implementation of tools and techniques to monitor conversion of sol-gel to solid state structures via electron, synchrotron radiation and scanning probe techniques.

\section{I.1.: Nanopatterning of Ceramic Oxides}

In order to explore the role of spatial/dimensional constraints on ceramic oxides, it was essential to develop the very approaches for nanopatterning of oxides. Based on continuous development of the soft$e \mathrm{BL}$ (Fig. 1) as an enabler for fabricating spatially and dimensionally constrained oxides, the research activities during the prior project focused on site-specific nanopatterning and microstructure evolution of dimensionally constrained oxide nanopatterns. We have demonstrated fine control over the internal microstructure and external morphology of the soft-eBL-prepared nanostructures. The controlled nanopatterns serve as test structures to help gain valuable insight into the structure-property relationship of functional oxides at miniaturized size scale. 
Figure 1: Schematic illustration of soft eBL nanopatterning approach for oxides, and associated advantages. SAM deposition

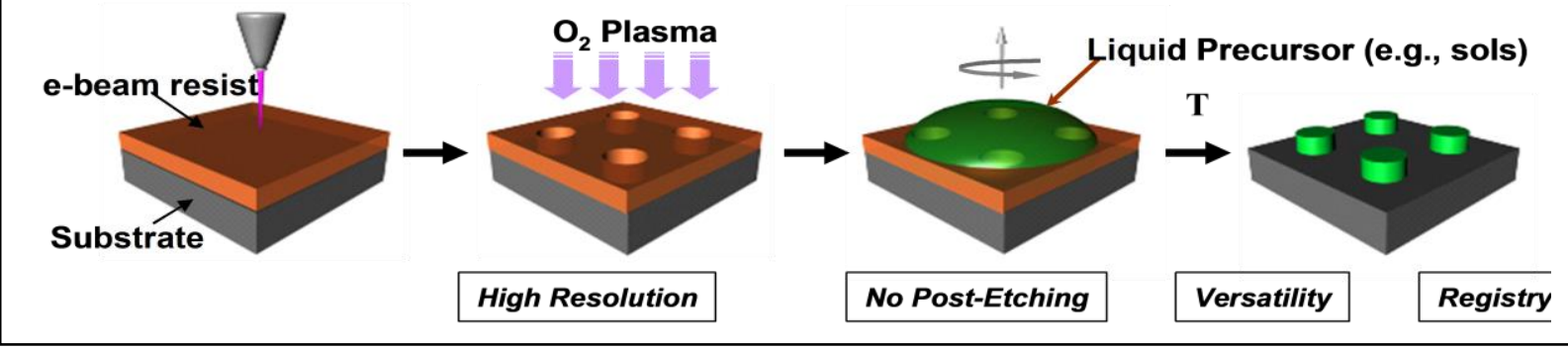

Among many notable prior highlights is the analytical development of so-called proximity parameters for soft eBL. Here, complex geometries such as "crosses" can be e-beam etched in resists by controlling the pattern geometry and dosage, as demonstrated in Figure 2, which shows that traditional geometry of "crosses" results in overexposure of "nodes", while optimized geometry results in remarkably uniform exposure, thus enabling nanopatterning of complex shapes.

\section{I.2: Microstructure Tailoring, Control and Versatility of Nanopatterned Oxides:}

Once patterned by soft eBL, the sol-gel structures are amenable to all the "tricks" of materials science to convert them to solid state form with thermal treatment regimen. Rapid thermal annealing (RTA) leads to rapid mass loss and shrinkage which is too fast to accommodate by diffusive process, leading to nanoporous structures. Slow and deliberate conversion on appropriate lattice and symmetry-matched substrate leads to conversion to their single crystal forms, while intermediate approach leads to controlled grain growth resulting in "beaded" or "bamboo" microstructures where grain boundaries are

Figure 2: Comparison of traditional eBL approach for "cross" patterns, using traditional geometry and exposure (Top). We have developed an analytical model for eBL exposure which relies on optimized geometry and pattern biasing, which allows uniform etching of e-resist resulting in uniform nanopatterns.

\section{Fabrication of BTO High Density} 2-D Patterns on STO

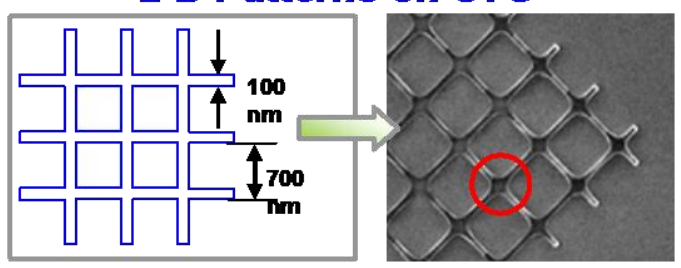

Overexposure at the Nodes

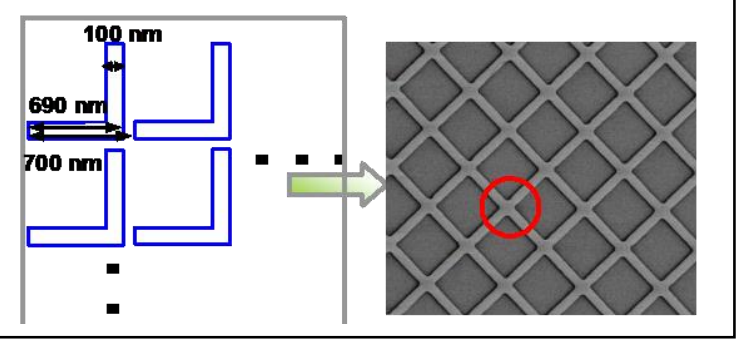
perpendicular to the long axis (Fig. 3).

We have applied this nanopatterning approach to many complex multifunctional oxides, ranging from simple $\mathrm{SnO} / \mathrm{ZnO}$ structures to multicomponent perovskites, spinels and magnetoplumbite structures. ${ }^{28-33}$ Further, it is remarkable that despite complex structures, unknown phase diagrams and chemical nonstoichiometry, and other variables, we have been able to optimize soft-eBL and thermal conditions for epitaxial conversion on appropriate strain- and symmetry-matched substrates.

The successful implementation of soft-eBL nanopatterned oxides has paved the way for not only understanding of the kinetics and thermodynamics of solid-state conversion of oxides under spatial and dimensional constraints, but has truly opened new vistas for probing the atomic- and nano-scale aspects of interfaces with their limited nanoscale length, and associated intricate influence on shape evolution of external morphology. 
Fig. 3: Tailoring of "internal" microstructure of "constrained" oxide nanopatterns.

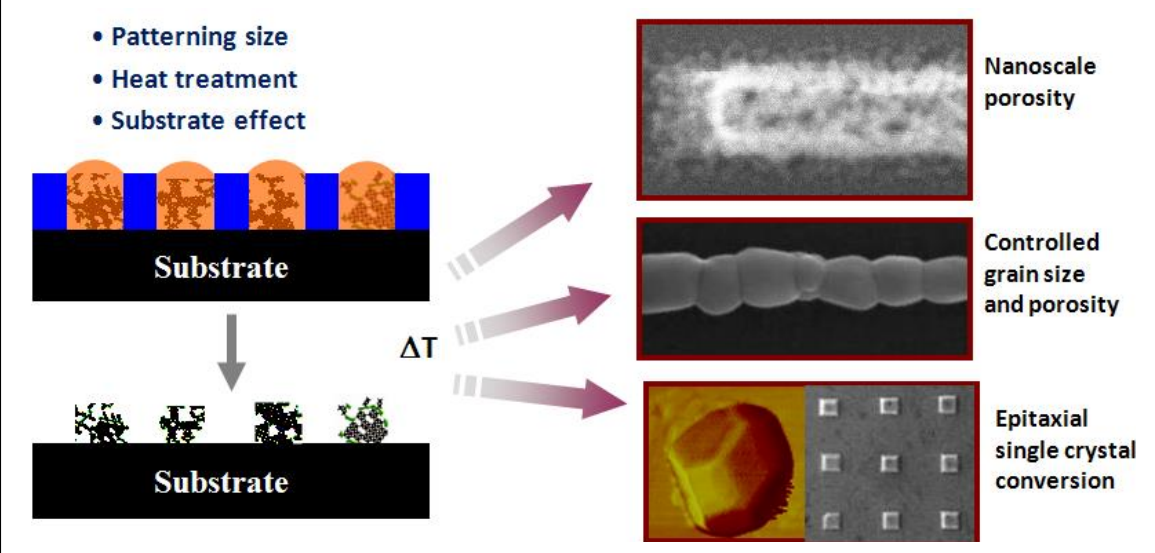

I.3. Characterization and Localized Properties of Nanopatterned Oxides:

Development of softeBL has, for the first time, provided an unprecedented access to nanopatterned oxides. There are a number of fundamental issues of nature and character of such constrained oxide nanopatterns, which were examined by a unique combination of

synchrotron radiation studies (at ANL-APS) and advanced electron and scanning probe microscopy.

Briefly, prior research accomplishments in regards to "characterization" include the following:

1) The evolution of microstructure of oxides from sol-gel state to solid-state form was monitored via in-situ x-ray scattering (GISAXS and GIWAXS). Particularly, the scientifically interesting and technologically relevant challenge of nanoscale porosity evolution was solved.

2) Synchrotron x-ray nanoprobe diffraction was utilized, for the first time, to probe the nature of interfacial strain between nanopatterns and substrates, with a particular noteworthy finding of edge effects in strain localization in nanopatterned oxides,

3) Scanning probe microscopy validated the functional character of nanopatterned oxides, thereby providing further credence that soft-eBL derived nanopatterned oxide do indeed display generally expected functional behavior, albeit modified due to spatial and dimensional constraints.

4) Part of the motivation to pursue nanopatterned oxides is to exploit the high surface area and locked-in microstructure in semiconducting metal oxides, for improved gas sensing performance. We have demonstrated that not only do nanopatterned oxides exhibit high sensitivity and selectivity (with dopants) but that they exhibit remarkably rapid response and recovery as well. Further, with the innovative use of UV exposure ${ }^{35}$ (to inject charge carriers), we have demonstrated unprecedented room temperature gas sensing ${ }^{36}$ behavior of soft-eBL derived nanopatterned oxides.

5) We also initiated real-time studies of nanopattern conversion with in-situ thermal TEM, both at Northwestern and at NCEM. The preliminary research has shown notable change in activation energy of grain growth in constrained oxides.

In the context of the specific ESPM program objectives, the prior research advanced our understanding of: a) charge mitigation in eBL with variable-pressure approach, b) analytical modeling of electron beam dosage for nanopatterning, c) combination of eBL with soft chemistry for nanopatterning of oxides which are often difficult to pattern owing to their chemical inertness and refractory nature, $d$ ) development of "gentle" soft-eBL, to enable nanopatterning on membranes and fragile substrates, which do not require further specimen preparation for subsequent analysis with electron, ion, photon and scanning probe microscopy/analysis, e) cross-correlative techniques encompassing synchrotron x-ray scattering (GISAXS, GIWAXS, nanoprobe diffraction) with advanced SEM, S/TEM and scanning probe microscopt, f) initiated synchrotron x-ray scattering (GISAXS, GIWAXS, nanoprobe) and electron microscopy for in-situ monitoring of phase conversion, g) demonstration of the efficacy of nanopatterned oxides as technological platform in gas sensing, at room temperature with potential for multiplexing; among other achievements. 


\section{I.4. Supported Personnel during the Prior Project: Graduated students}

1. Ms.(Dr.) Zixiao Pan: MSE, PhD, Spring 2008 - now at Exponent, CA.

2. Mr. (Dr.) Suresh Donthu: MSE, PhD, Summer 2008 - now Exponent, MD.

3. Mr. Tao Sun: MSE PhD, Fall 2009 - now at ANL, APS Director's postdoctoral fellowship (w/Dr. J. Murray Gibson).

4. Mr. Ben Myers: MS in MSE, Fall 2006 - now a staff scientist NUANCE Center, NU.

5. Ms. Shanwei Fan: MS in MSE, Summer 2009; now a research engineer, Taiwan Semiconductors, Taiwan.

6. Mr. Ken D'Aguila: BS in MSE (UG senior thesis student), Fall 2008 - now a PhD graduate student (w/Amanda Petford-Long) in MSE, NU.

\section{Ongoing/current graduate students:}

7. Ms. Aiming Yan: $3^{\text {rd }}$ year PhD student: Aiming is continuing in-situ S/TEM of nucleation and growth of nanopatterned oxides, and has an active proposal access to NCEM facilities. Further, having developed considerable experience (and expertise) in specialized S/TEM techniques, she is poised to undertake 3-D tomographic measurements of various stages of nucleation/growth as well as shape evolution of nanopatterned oxides.

8. Mr. Yi-Kai Huang: $2^{\text {nd }}$ year PhD student: Yi-Kai is developing innovative nanopatterning geometries for oxides and localized measurements with advanced S/TEM, particularly FIB specimen preparation, followed by HRTEM, STEM HAADF imaging and spectroscopy. He will continue that work as well as collaborate with Drs. Tao Sun and Murray Gibson on broad-beam quantitative characterization with synchrotron x-ray scattering (SAXS/WAXS, nanoprobe).

9. Mr. Bin Liu: $3^{\text {rd }}$ year PhD student; jointly with ANL-MSD (Drs. Amanda Petford-Long and Seubung Hong): Bin has focused his thesis research on innovative SPM techniques for probing localized dynamic properties of oxide nanopatterns, including polarization and magnetization measurements. Bin is stationed at ANL under CNM program, and will advance the dynamic SPM aspects of proposed research.

I.5. Archival Publications: Published/in-press or in-preparation: Peer reviewed archival publications primarily from current DOE-BES project support:

1) Pan Z, Li S, Wang Z Yu MF, V.P. Dravid, ,'Patterning-controlled morphology of spatially and dimensionally constrained oxide nanostructures", Applied Physics Letters Vol: 91(14) 143105 (2007)

2) Method of Making Nanopatterns And Nanostructures And Nanopatterned Functional Oxide Materials, NU Invention Disclosure, \# 25069, 2007, Vinayak Dravid, Suresh Donthu, Zixiao Pan, 2007. U.S. Patent Application \# 20080070010, 20 March 2008.

3) T. Sun, H. Hu, Z. Pan, X. Li, J. Wang and Vinayak P. Dravid, "In-situ real-time investigation of kinetics of nucleation and growth of sol-gel-derived functional oxide thin films", Phys. Rev. B 77, 205414 (2008)

4) Muto A, Buchholz D, Chang, R, Dravid, VP, "Multimodal Imaging of Nanostructures with FEG SEM", Microscopy \& Microanalysis, Vol. 14 (2): pp: 686-7 (2008)

5) Myers B, Pan Z, Dravid VP, "Beam skirting effects on energy deposition profile in VP-SEM", Microscopy and Microanalysis, Vol.14 (2): pp: 1208-9 (2008)

6) Donthu S, Alem N, Pan Z, Li SY, Shekhawat G, Dravid V, Benkstein KD, Semancik $S$, "Directed fabrication of ceramic nanostructures on fragile substrates using soft- 
electron beam lithography (soft-eBL)", IEEE Trans on Nanotech, Vol. 7 (3): pp. 338343 (2008)

7) Review/highlight publication: Dravid VP, "Controlling" Internal Microstructure of Nanopatterned Oxides vis Sof Electron Beam Lithography (Soft-eBL)", Journal of Materials Chemistry, Vol. 19 (25): pp: 4295-4299: DOI: 10.1039/B903201K (2009)

8) Aiming Yan and Vinayak P. Dravid, "In-situ TEM Heating Studies of Nucleation and Growth of Nanopatterned Oxides", submitted, Microscopy and Microanalysis, 2010.

9) Bin Liu and Vinayak P. Dravid, "Giant and Sharp Magnetoferroelectric Effect in Sol-Gel Derived Nanocomposites", submitted to NanoLetters, 2010.

10) Tao Sun, Jin Wang and Vinayak P. Dravid, "Localized Strain Measurements via Diffuse X-ray Scattering of Nanopatterned Oxides"; under-review, Physical Review B, 2010.

11) Xie Sujing, Sterbinsky George, Wessels, Bruce, Dravid Vinayak, "Defect and interfacial structure of heteroepitaxial Fe3O4/BaTiO3 bilayers", Microscopy and Microanalysis, submitted August (2009), accepted for publication (2010).

\section{Peer reviewed archival publications with partial support from the DOE-BES project}

13. Sun T,Pan ZX, Dravid VP, Wang, ZY, Yu, MF, Wang, J, "Nanopatterning of Multiferroic $\mathrm{BiFeO}_{3}$ Using "Soft" Electron Beam Lithography", Applied Physics Letters Vol. 89 (16): pp: 163117 (2006)

14. Pan ZX, Alem N, Sun T, Dravid VP, "Site-specific Fabrication and Epitaxial Conversion of Functional Oxide Nanodisk Arrays”, Nano Letters Vol. 6 (10): pp: 2344-2348 (2006)

15. Donthu S, Sun T, Dravid VP, "Fabrication and Structural Evaluation of Beaded Inorganic Nanostructures Using Soft Electron-Beam Lithography", Advanced Materials Vol. 19 (1): pp: 125 (2007)

16. Mundra MK, Donthu SK, Dravid VP, Torkelson JM, "Effect of spatial confinement on the glass-transition temperature of patterned polymer nanostructures", Nano Letters Vol. 7 (3): pp: 713-718 (2007)

17. Pan Z, Li S, Wang Z Yu MF, Dravid VP, "Patterning-controlled morphology of spatially and dimensionally constrained oxide nanostructures", Applied Physics Letters Vol. 91(14): pp: 143105 (2007)

18. Sun, T., Hu, H., Pan, Z., Li, X., Wang, J., Dravid, V.P., "In situ real-time investigation of kinetics of nucleation and growth of sol-gel-derived functional oxide thin films", Physical Review B. 77 (20), 2008: 205414

19. Xie, S, Cheng J., Wessels BW, Dravid, V. P. , "Interfacial structure and chemistry of epitaxial CoFe2O4 thin films on $\mathrm{SrTiO} 3$ and $\mathrm{MgO}$ substrates", Appl. Phy. Lett., Vol. 93, Issue 18, Article No.: 181901 (2008)

20. Klajn R, Gray TP, Wesson PJ, Myers BD, Dravid VP, Smoukov SK, Grzybowski BA, "Bulk synthesis and surface patterning of nanoporous metals and alloys from suprspherical nanoparticle aggregates", Advanced Functional Materials, Vol. 18 (18): pp: 2763-2769 (2008)

21. Sun, T., Donthu, S., Sprung, M., D'Aquila, K., Jiang, Z., Wang, J., Dravid, V.P., "Effect of Pd doping on the microstructure and gas sensing performance of mesoporous tin oxide thin films", Acta Materialia, Vol. 57, Issue 4, pp.: 1095-1104 (2009) 
22. Dravid Vinayak P, "Nano: The Big Way Forward", The Economic Times - Polymers Vol. 5: pp: 21-28 (2008-2009)

\section{Honors/Awards \& Noteworthy Activities:}

\section{Vinayak P. Dravid, PI:}

1. Inauguration/inception class of fellows: Microscopy Society of America (MSA), 2009

2. Richard M. Fulrath Award: American and Japanese Ceramic Societies, 2008

3. Election to Faculty Honor Roll: Northwestern University: Selected by voting UG students for excellence in teaching; 2009

4. First McCormick Faculty Excellence Award: Northwestern University; 2006

5. Among several invited talks, two "Keynote Addresses" include one at: Sanken International Symposium, Osaka University, Japan, 2009; and ICONSAT 2010, IIT Bombay, India, 2010.

6. Member, Working Group; NNI beyond 2010; Chicago, IL, 2010, a workshop under US NNI Program to assess opportunities for next decade of NNI.

7. Review committee, DOE-BES Electron Beam Microcharacterization Centers (EBMC), ORNL and NCEM: 2008, 2009, 2010; Review Committee, Program Review of MSD, NCEM.

\section{Recognition to Students:}

1) Mr. S. Donthu: American Vacuum Society outstanding graduate student award, 2007

2) Ms. Z. Pan: First prize, InNUvation Applied Research Day (business plan contest Northwestern University), 2007

3) Mr. Tao Sun:

i. Outstanding graduate student award, NU, 2008

ii. First Place award: "Array of Mount Doon”, International Materials Research Conference, Chongqing, PRC, October 2009.

iii. Director's postdoctoral fellowship, ANL-APS (w/J. Murray Gibson), 2009.

4) Ms. Aiming Yan and Mr. Bin Liu:

i. ASU CHREM student scholarships, January 2009 